Full Length Article

\title{
OBP fused with cell-penetrating peptides promotes liposomal transduction
}

 \\ Artur Ribeiro $^{a}$, Artur Cavaco-Paulo ${ }^{a, *}$ \\ a Centre of Biological Engineering, University of Minho, Campus de Gualtar, 4710-057, Braga, Portugal \\ b 3Bís Research Group, Biomaterials, Biodegradables and Biomimetics, University of Minho, Headquarters of the European Institute of Excellence on Tissue \\ Engineering and Regenerative Medicine, Ave Park, 4806-909 Taipas, Guimarães, Portugal \\ ' ICVS/3B's, PT Government Associate Laboratory, Braga, Guimarães, Portugal
}

\section{A R T I C L E I N F O}

\section{Article history:}

Received 22 May 2017

Received in revised form 2 November 2017

Accepted 8 November 2017

Available online 10 November 2017

\section{Keywords:}

1-aminoanthracene (1-AMA)

Cell-penetrating peptides

Odorant-binding protein

1-AMA transduction

Liposomes

\begin{abstract}
A B S T R A C T
Cell-penetrating peptides (CPPs) have been applied as novel transport systems with the ability to facilitate the delivery of peptides, proteins, and oligonucleotides into cells. Herein, we designed different fusion proteins composed by pig odorant binding protein (OBP-I) and three CPPs, namely Tat, pVEC and Pep-1. A new methodology using liposomes as reservoirs and OBP:CPPs as carriers was developed as an advanced system to capture odorant molecules. 1-aminoanthracene (1-AMA) was used as a model molecule to evaluate the transduction ability of OBP:CPPs into the reservoirs. The transduction efficiency was dependent on the initial capacity of OBP:CPPs to bind 1-AMA and on the penetration of liposomes promoted by the CPPs. An encapsulation efficiency of $42 \%$ was obtained with OBP:Tat fusion protein. The presence of Tat peptide increased the 1-AMA transduction of 1.3 and 2.5 fold compared with Pep-1 and pVEC, respectively. This work expands the application of OBPs and CPPs on the design of promising capture and delivery systems for textile and cosmetic applications.
\end{abstract}

(C) 2017 Elsevier B.V. All rights reserved.

\section{Introduction}

CPPs are cationic peptides, normally up to 30 residues, which can be amphipathic or hydrophobic, possessing low cellular toxicity [1]. These peptides are widely studied to deliver biologically active molecules into cells, such as peptides, proteins, RNA, DNA, oligonucleotides and liposomes without the need of specific membrane receptors [1-3]. Several works report the use of CPPs in biomedical applications. Tat-conjugated quantum dots administrated intraarterially at a proximal cervical carotid artery in rats were able to cross the highly impermeable blood-brain barrier [4]. Elmquist et al. (2001) demonstrated the internalization of pVEC labeled with fluorescein isothiocyanate (FITC) into three different endothelial cell lines after treatment with this peptide [5]. Jing et al. (2016) demonstrated that the combined use of CPPs-loaded nanobubbles with ultrasound-targeted microbubble destruction (UTMD) technology could efficiently improve gene transfection in cultured breast cancer TNBC cells [6]. The application of CPPs in other areas rather than therapeutics, such as textile functionalization, was

\footnotetext{
* Corresponding author.

E-mail address: artur@deb.uminho.pt (A. Cavaco-Paulo).
}

exploited herein for the first time. CPPs were conjugated with an odorant-binding protein (OBP) for the capture and transduction of odorant molecules. Liposomes were used as final reservoirs and 1-AMA as the model molecule.

Odorant-binding protein I (PDB 1DZK code) is a transport protein present in the nasal mucosa of pig constituted by 157 amino acids. This protein was selected for this study based on the information available about the three-dimensional structure and the binding specificity for a large number of natural and synthetic molecules $[7,8]$. OBP-I is composed by eight antiparallel $\beta$-sheets [9], forming an internal cavity to bind different ligands, like terpenoids, aromatic compounds, aliphatic molecules and aldehydes [7]. OBPs have been studied in several applications. Wei et al. (2008) designed different mutants of pig OBP to bind several aromatic polycyclic hydrocarbons. This study opened the view for the use of OBPs as biosensors for the monitoring of aromatic pollutants [10]. Di Pietrantonio et al. (2013) used surface acoustic wave (SAW) biosensor systems with three different OBPs as probes for the detection of low concentration of octanol (13 ppm) and carvone (9 ppm) [11]. More recently, Silva et al. (2014) used pig OBP for the reduction of unpleasant odors and controlled release of fragrances when immobilized in fabric supports [12]. 

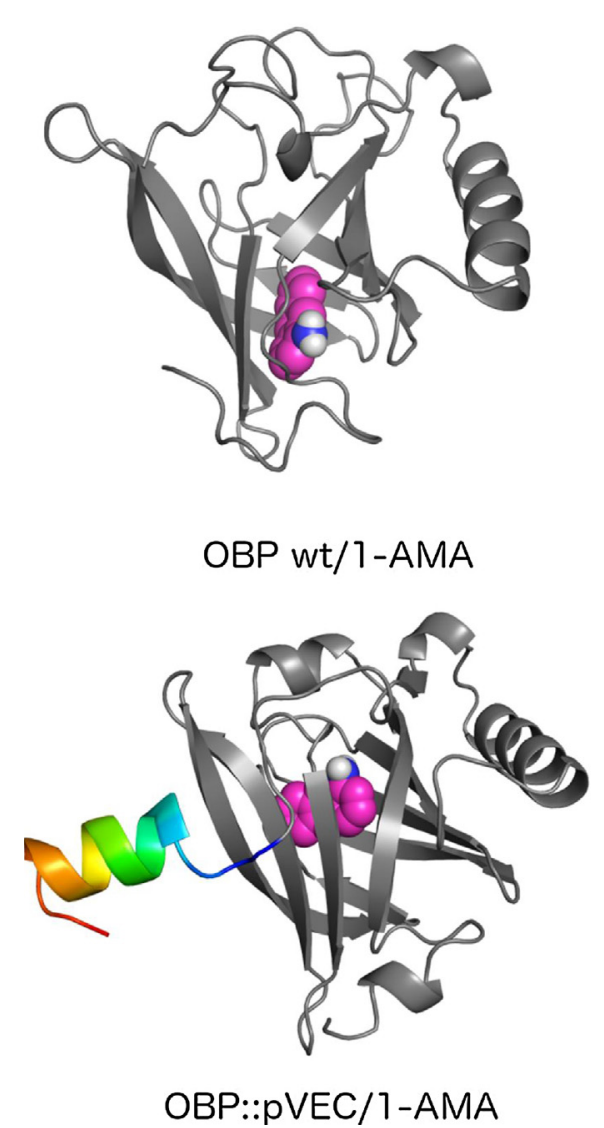
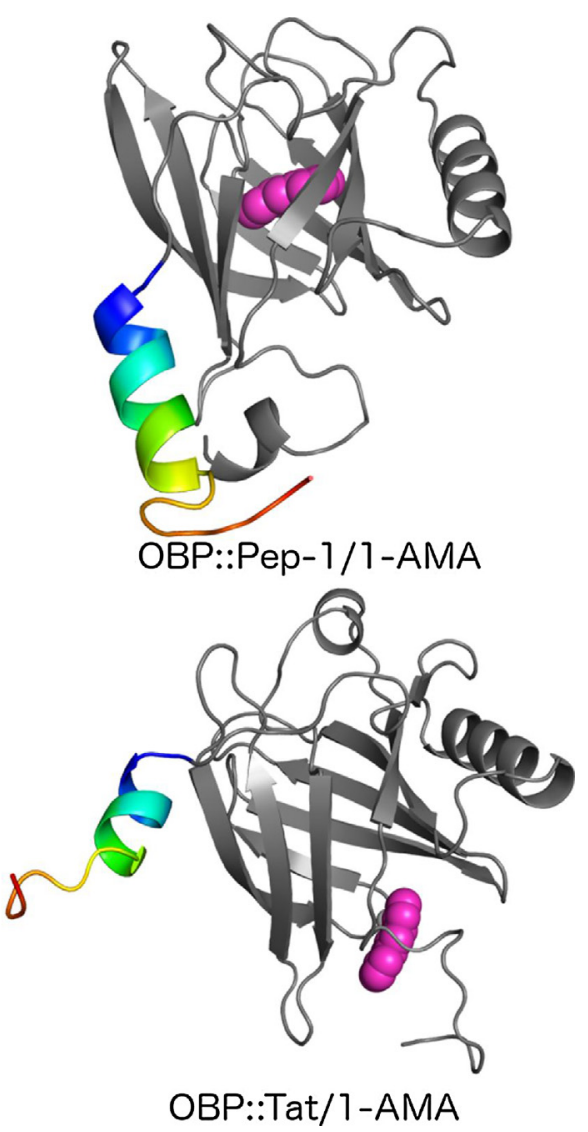

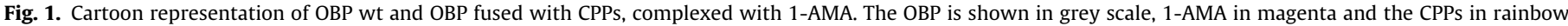

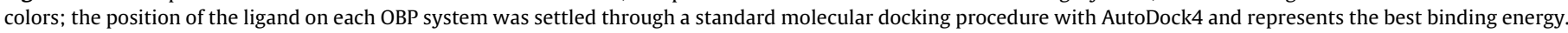

We designed for the first time three OBP:CPPs to be used as carriers and transducers of a model molecule, 1-aminoanthracene (1-AMA), as represented in Fig. 1. The CPPs used in this work were Tat, pVEC and Pep-1. Tat corresponds to the basic domain of HIV-1 Tat protein rich in arginine residues [13]. pVEC is derived from the murine sequence of the cell adhesion molecule vascular endothelial cadherin with an amphipathic character [5] and Pep-1 is a synthetic peptide that belongs to the group of amphipathic peptides. It contains a hydrophobic tryptophan-rich domain and a hydrophilic lysine-rich domain [14]. 1-AMA is the most used ligand to characterize the ligand binding properties of OBP family members $[15,16]$. When 1-AMA binds to OBP-I, the maximum wavelength of AMA$\mathrm{OBP}$ complex is shifted from 537 to $481 \mathrm{~nm}$, with an increase of the fluorescence intensity compared with the ligand alone $[9,12]$.

A new methodology was developed to evaluate the transport and transduction of 1-AMA into reservoirs promoted by OBP:CPPs.

\section{Materials and methods}

\subsection{Reagents}

Tris-base, imidazole, sodium phosphate and sodium chloride were obtained from Sigma-Aldrich. 1-AMA was purchased from TCI chemicals. Nickel Magnetic Beads for His Tag Protein Purification was available from Biotool, Bimake and Molecular weight Precision Plus Protein ${ }^{\mathrm{TM}}$ standards were purchased from BioRad. Culture medium was purchased from GRISP. 1,2-Dioleoylsn-glycero-3-phosphoethanolamine (DOPE), and 1,2-distearoyl-snglycero-3-phosphoethanolamine-N-[amino(polyethylene glycol)2000] (DSPE-PEG) were purchased from Lipoid. Cholesterol was acquired from Sigma-Aldrich. Fluorescein isothiocyanate (FITC) was acquired from Sigma-Aldrich and Alexa Fluor 467 was purchased from ThermoFisher Scientific. All other reagents were acquired from Sigma-Aldrich and used as received.

\subsection{OBP fusion constructs}

Three CPPs: Tat (GRKKRRQRRRPPQ), Pep-1 (KETWWETWWTEWSQPKKKRKV) and PVEC (LLIILRRRIRKQAHAHSK) were fused in the C-terminus of OBP-I. Gene sequences (OBP:Tat, OBP:Pep-1 and OBP:pVEC) were synthetized by GenScript and cloned in pET-28a plasmid.

\subsection{CPPs synthesis}

CPPs were synthetized by JPT peptide technologies GmbH with 94.7\%, $98.0 \%$ and $98.2 \%$ of purity for Tat, Pep- 1 and pVEC, respectively. These peptides were used as experiment controls.

\subsection{Expression and purification of fusion OBPs}

Escherichia coli BL21(DE3) harboring the pET-28a:OBP-CPPs was used for protein expression in Terrific Broth (TB) medium supplemented with lactose. Cells were harvested by centrifugation at $7000 \mathrm{~g}$, for $5 \mathrm{~min}$ at $4{ }^{\circ} \mathrm{C}$ and resuspended in phosphate buffer ( $20 \mathrm{mM}$ sodium phosphate, $500 \mathrm{mM} \mathrm{NaCl}, \mathrm{pH} 7.4$ ) supplemented with $10 \mathrm{mM}$ of imidazole and lysed by sonication (50\%, 3.0 s ON, $9.0 \mathrm{~s}$ OFF for $10 \mathrm{~min}$ ) in sonicator vibracell ${ }^{\mathrm{TM}}$ SONICS. Soluble and insoluble fractions were separated by centrifugation at $12,000 \mathrm{~g}$, for $30 \mathrm{~min}$ at $4{ }^{\circ} \mathrm{C}$. The soluble fraction was purified through Nickel magnetic beads with specificity to His-tag present in the protein's $\mathrm{N}$-terminal. The purity of OBP:CPPs was evaluated by Sodium Dode- 
cyl Sulfate Polyacrylamide Gel Electrophoresis (SDS-PAGE) under reducing conditions. To remove the presence of salts and imidazole after purification the samples were dialyzed for 3 days, at $4{ }^{\circ} \mathrm{C}$ against ultrapure water.

\subsection{Characterization of OBP:CPPs proteins}

\subsubsection{SDS-PAGE gel electrophoresis}

Lyophilized proteins were solubilized in $50 \mathrm{mM}$ Tris- $\mathrm{HCl} \mathrm{pH} 7.5$, loaded on SDS-PAGE gel and stained with Coomassie solution to analyze size and purity.

\subsubsection{MALDI-TOF mass spectrometry}

Mass/charge of OBP fusion proteins was verified by MatrixAssisted Laser Desorption/Ionization with time-of-flight (MALDITOF) using sinapic acid (SA) as the matrix ( $\geq 99.5 \%$ ). The mass spectra were acquired on an Ultra-flex MALDI-TOF mass spectrophotometer (Bruker Daltonics $\mathrm{GmbH}$ ) equipped with a $337 \mathrm{~nm}$ nitrogen laser. A double layer deposition was used to analyze OBP fusion proteins. For this, a saturated solution of SA in ethanol was deposited in the ground steel plate until dry. Each sample, previously dissolved in TA30 (30\% acetonitrile/70\% TFA), was mixed (1:1) with a saturated solution of SA in TA30. A volume of $2 \mu \mathrm{L}$ of each mixture was spotted onto the ground steel target plate (Bruker part $\left.n^{\circ} 209519\right)$ and analyzed using the reflective positive mode.

\subsubsection{Circular dichroism spectroscopy}

The structural state of OBP:CPPs in the presence or absence of ligand (1-AMA) was investigated by circular dichroism (CD) spectroscopy, using a Jasco J-1500 spectropolarimeter equipped with a temperature controller. The structure of CPP peptides was also analyzed. $\mathrm{CD}$ spectra were recorded at $37^{\circ} \mathrm{C}$, using $10 \mu \mathrm{M}$ as a fixed concentration for all the samples tested. The spectra were obtained over the wavelength interval of $180-260 \mathrm{~nm}$ at a scan speed of $20 \mathrm{~nm} / \mathrm{min}$ and bandwidth of $1 \mathrm{~nm}$. The path-length cell was $1 \mathrm{~mm}$. Baseline was recorded with the same buffer of the samples (phosphate buffer, $\mathrm{pH}$ 7.5) and subtracted to the protein spectra. Final spectra were obtained by the average of three scans for each sample.

\subsubsection{Fluorescence binding assays}

The binding capacity of wild-type OBP-I (OBP wt) and OBP:CPPs was determined by direct titration with 1-AMA, as reported in Silva et al. (2013) [12]. Briefly, the fluorescent probe, 1-AMA, was dissolved in $95 \%$ ethanol at $1 \mathrm{mM}$. Increasing ligand concentrations were added to $1 \mu \mathrm{M}$ of each protein in $50 \mathrm{mM}$ Tris- $\mathrm{HCl}, \mathrm{pH} 7.5$ and incubated at $37^{\circ} \mathrm{C}$ for $1 \mathrm{~h}$. Fluorescence emission spectra were recorded measuring the OBP-ligand complex formation by increase in the emission intensity at $481 \mathrm{~nm}$ when excited at $295 \mathrm{~nm}[12,17]$. Measurements were recorded in triplicate, on a microplate spectrofluorometer (BioTek Synergy MX) equipped with a temperature controller. Dissociation constants (Kd) were calculated from a plot of fluorescence intensity versus concentration of ligand, obtained with a standard non-linear regression method, described in Malpeli et al. (1998) [18].

\subsection{Liposomes preparation}

Liposomes $(18 \mathrm{mM})$ were prepared based in a formulation developed previously in our group, using $52.5 \%$ of 1,2-Dioleoyl-sn-glycero-3-phosphoethanolamine (DOPE), $42.5 \%$ of cholesterol (CHOL) and 5\% of 1,2-distearoyl-sn-glycero-3phosphoethanolamine-N-[amino(polyethylene glycol)-2000] (DSPE-PEG) through ethanol injection method [19]. Further, the liposomes were extruded through $200 \mathrm{~nm}$ polycarbonate filters
(Nucleopore) followed by several passages through $100 \mathrm{~nm}$ polycarbonate filters (Nucleopore) to reduce the size of the vesicles, using an extruder supplied by Lipex Biomembranes Inc. The size distribution and surface charge of liposomes were characterized by dynamic light scattering (DLS).

\subsection{Transduction of 1-AMA into liposomes reservoirs}

To determine the concentration of 1-AMA transduced to liposomes, a new procedure was designed and implemented (Fig. 2). At step $1,100 \mu \mathrm{M}$ of OBP:CPPs were incubated with $100 \mu \mathrm{M}$ of 1 AMA ligand for $60 \mathrm{~min}$ at $37^{\circ} \mathrm{C}$ to promote binding. The free 1-AMA was separated from OBP:CPPs/1-AMA complex by size exclusion chromatography using a PD-10 Desalting Column, with Sephadex G-25 resin (GE-Healthcare; cut-off $5 \mathrm{kDa}$ ). Afterward, in step 2, the OBP:CPP/1-AMA complex was incubated with liposomes (at a final concentration of $14.4 \mathrm{mM}$ ) for $60 \mathrm{~min}$ at $37^{\circ} \mathrm{C}$. Liposomes containing 1-AMA were separated from the complex by Vivaspin ${ }^{\circledR}$ 500 centrifugal filter tubes (cut-off $100 \mathrm{kDa}$ ). 1-AMA in the presence of liposomes (without protein) was performed as a control on the liposomal transduction experiments. The 1-AMA transduction efficiency was calculated using Eq. (1):

$\mathrm{TE}_{(1-\mathrm{AMA})}=\left[1-\left(\frac{\text { Free } 1-\text { AMA }}{1+\text { Free } 1-A M A_{2}}\right)\right] \times 100$

where $\mathrm{TE}_{(1-\mathrm{AMA})}$ is the transduction efficiency (\%) of 1-AMA into liposomes; Free $1-\mathrm{AMA}_{1}$ is the concentration of 1-AMA measured by fluorescence in step 1 ; Free $1-\mathrm{AMA}_{2}$ is the concentration of 1 AMA measured by fluorescence in step 2; total 1-AMA is the initial concentration added to the system.

The concentration values of 1-AMA were determined by measuring the fluorescence of free 1-AMA fractions in step 1 and in the step 2 at $600 \mathrm{~nm}\left(\lambda_{\mathrm{ex}}=295 \mathrm{~nm}\right)$ and substituting the value in the equation obtained from a calibration curve of fluorescence versus 1-AMA concentration.

The effect of CPP on 1-AMA transduction was determined using Eq. (2):

$\mathrm{CPP}_{\text {effect }}=\mathrm{TE}_{\mathrm{OBP}: \mathrm{:CPP}}-\mathrm{TE}_{\mathrm{OBP} w \mathrm{wt}}$

where $\mathrm{TE}_{\mathrm{OBP}: \mathrm{CP}}$ is the transduction efficiency calculated for $\mathrm{OBP}: \mathrm{CPP}$ and $\mathrm{TE}_{\mathrm{OBPwt}}$ is the transduction efficiency determined for wild-type OBP, both using equation 1.

Measurements were recorded in two independent experiments and the results were expressed as mean value \pm standard deviation (SD).

After transduction, 1-AMA is expected to be found in different locations of the system. In order to evaluate its localization and distribution, additional fluorescence spectra were obtained and analyzed using the protocol described in the supplementary material.

The concentration of OBP:CPP conjugated with liposomes after 1-AMA transduction was determined by measuring the absorbance at $280 \mathrm{~nm}$. The free protein was separated from the conjugate (OBP:CPP/liposome) using a $100 \mathrm{kDa}$ ultrafiltration membrane. The amount of protein (\%) conjugated was calculated according to Eq. (3):

$\mathrm{OBP}:: \mathrm{CPP}_{\text {conjugated }}=\left(\frac{\mathrm{OBP}:: \mathrm{CPP}_{\text {initial }}-\mathrm{OBP}:: \mathrm{CPP}_{\text {free }}}{\mathrm{OBP}:: \mathrm{CPP}_{\text {initial }}}\right) \times 100$

\subsection{Dynamic light scattering}

The mean size diameter ( $\mathrm{nm}$ ), polydispersity index (PDI) and zeta-potential $(\mathrm{mV})$ of the liposomes were measured in a Zetasizer Nano ZS (Malvern Instruments) at $25^{\circ} \mathrm{C}$.

Prior to DLS measurements, the samples were diluted with PBS at $\mathrm{pH} 7.4$ (for size) and with ultrapure water (for zeta-potential). All 


\section{EXPERIMENTAL SETUP}

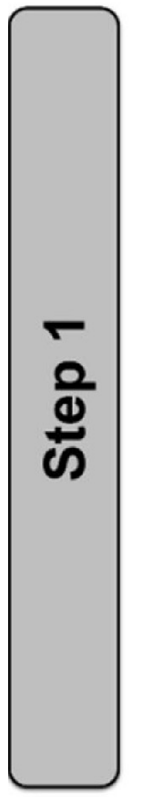

\section{$100 \mu \mathrm{M}$ OBP::CPP + $100 \mu \mathrm{M}$ 1-AMA}
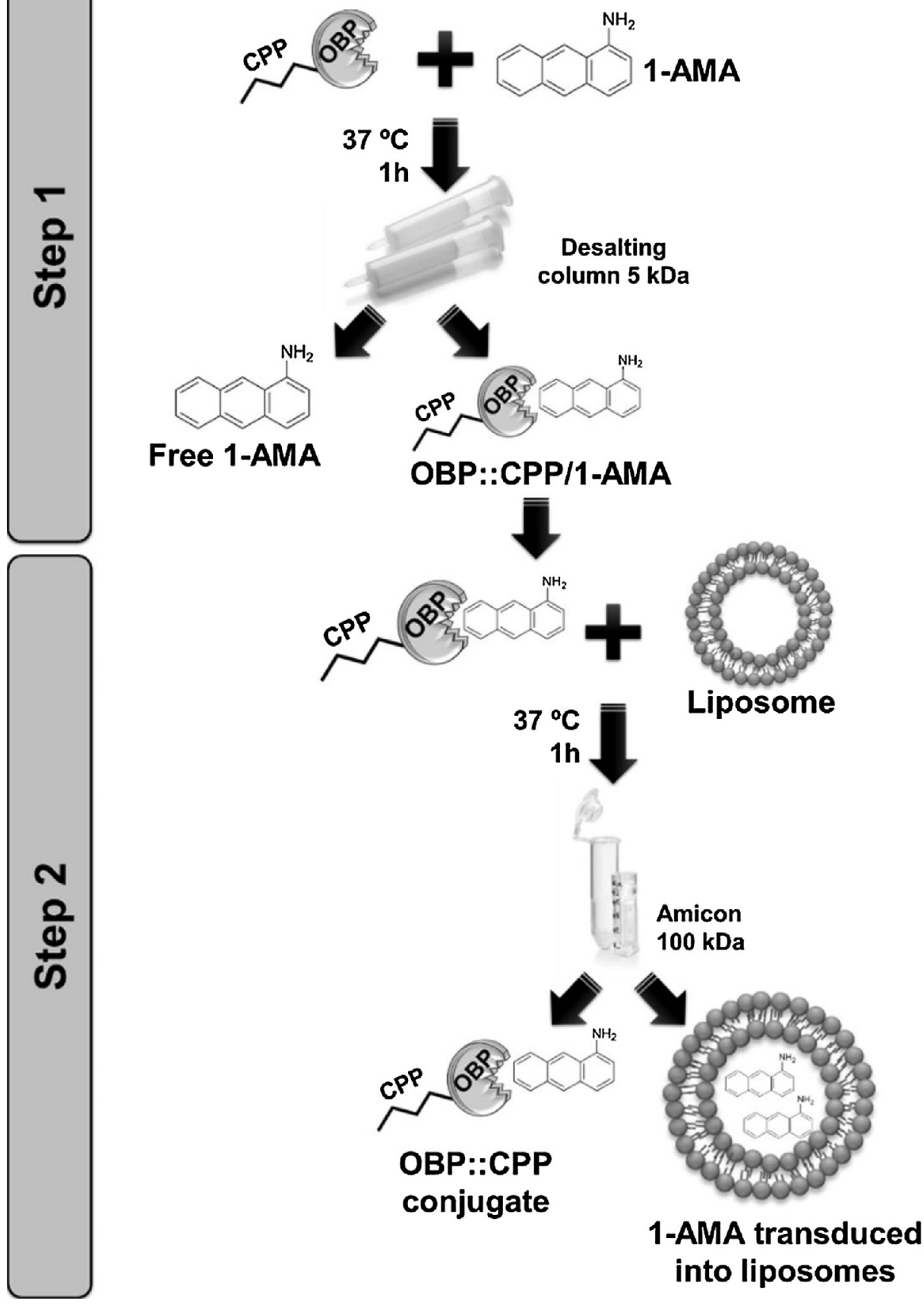

Fig. 2. Experimental procedure to evaluate the 1-AMA transduction into liposomes.

measurements were read in triplicate, being the results described as mean \pm standard deviation.

\subsection{Molecular dynamics and free energy calculations}

We evaluated the delivery of 1-AMA by OBP wt to a lipid bilayer model, through molecular dynamics (MD) simulations. For this, we simulated independently the complex OBP/1-AMA (Fig. S1) and a membrane containing 200 lipids (Fig. S2), with the same experimental proportion of DOPE (53\%), DSPE (5\%) and CHOL (42\%). After the equilibration of the two systems mentioned above, umbrella sampling (US) simulations were carried out to estimate the free energy involved in the unbinding process and delivery of the 1AMA in the membrane (Fig. S3). Parameters and calculation options for MD simulations on complex and membrane are detailed in Supplementary Data. The X-ray structure of OBP used in this study is available on the Protein Data Bank [20], with the code 1DZK [21].

We designed with the program PyMOL [22] a simulation box containing the bilayer and the complex, which was centralized and positioned with the opening of the active channel facing the mem- 
Table 1

Amino acid sequence, molecular weight and ionic charge of CPPs.

\begin{tabular}{|c|c|c|c|c|}
\hline $\mathrm{CPP}$ & Amino acid sequence & Molecular weight $(\mathrm{Da})^{\mathrm{a}}$ & Total charge $^{\mathrm{a}}$ & Structure $^{b}$ \\
\hline Tat & GRKKRRQRRRPPQ & 1719.04 & +8 & \\
\hline pVEC & LLIILRRRIRKQAHAHSK & 2209.72 & +6 & 3 \\
\hline Pep-1 & KETWWETWWTEWSQPKKKRKV & 2848.26 & +3 & \\
\hline
\end{tabular}

b De novo peptide structure prediction in http://mobyle.rpbs.univ-paris-diderot.fr/cgi-bin/portal.py\#forms:PEP-FOLD3.

brane. Simple Point Charge (SPC) water model was used to solvate the system, in a box with $9.8 \times 9.8 \times 17.7 \mathrm{~nm}$ of dimension, making a large water layer above and below the lipid bilayer. Thus, the system had about 41000 water molecules. Due to the protein charged character, which has several residues deprotonated at physiological conditions, we add $19 \mathrm{Na}^{+}$ions until the box had zero net charge.

All simulations were performed using the GROMACS 4.5 .4 version [1,23], within the GROMOS 54a7 force field (FF) [24]. One stage of energy minimization was performed using a maximum of 12000 steps with steepest descent algorithm, then the system was initialized with the Lennard-Jones interactions truncated at $1.4 \mathrm{~nm}$ and using particle-mesh Ewald (PME) [25] method for electrostatic interactions, also with a cut-off of $1.4 \mathrm{~nm}$. The algorithm LINCS $[26,27]$ was used to constrain the chemical bonds of the peptides and the algorithm SETTLE [28] in the case of water. The pressure and temperature Berendsen algorithms [29] were used to control the temperature and pressure at $310 \mathrm{~K}\left(\approx 37^{\circ} \mathrm{C}\right)$ and $1 \mathrm{~atm}$, respectively. We used the following coupling constants: $\tau_{\mathrm{T}}=0.10 \mathrm{ps}$ and $\tau_{\mathrm{P}}=2.0 \mathrm{ps}$. Position restraints (with force constant of $1000 \mathrm{~kJ}$ $\& 903 \mathrm{~mol}^{-1} \& 903 \mathrm{~nm}^{-2}$ ) were applied to all protein heavy atoms and lipids for energy minimization and initialization (200 ps).

After this procedure, we run $1 \mathrm{~ns}$ of pulling MD simulation, with an integration interval of $1 \mathrm{fs}$, to generate the configurations needed for the US. In this step, a pull force was applied to move the 1-AMA ligand in the $\mathrm{z}$ direction, causing the OBP unbinding and entering on the model membrane, in a pull coordinate rate of $0.01 \mathrm{~nm} / \mathrm{ps}$. The protein was position restrained to fix its position and move only the 1-AMA. At this stage, Nose-Hoover [30,31] algorithm was used to couple temperature at $310 \mathrm{~K}$ and semi isotropic ParrinelloRahman barostat [32] for pressure coupling at $1.0 \mathrm{~atm}$. We used the following coupling constants: $\tau_{\mathrm{T}}=0.5 \mathrm{ps}$ and $\tau_{\mathrm{P}}=1.0 \mathrm{ps}$.

The potentials of mean force (PMF) were calculated using a combination of US [33-35] and the weighted histogram analysis method (WHAM) [33,36], on the configurations generated in the previous step. A spacing of $0.2 \mathrm{~nm}$ was used in the direction normal to the bilayer, resulting in 30 US simulations to sample the reaction coordinate; about $6 \mathrm{~nm}$ in the $z$ direction from the start position to the membrane center of mass. Each window was initialized for $100 \mathrm{ps}$ and simulated during $1 \mathrm{~ns}$. The harmonic biasing potential effects were removed using the weighted histogram analysis method after discarding the first 100 ps as equilibration.

\section{Results and discussion}

The designed fusion proteins comprised OBP-I protein and three cationic CPPs, namely Tat, pVEC and Pep-1 (Table 1).

\subsection{Purity and size of fusion proteins}

The three OBP:CPP fusion proteins were characterized regarding purity and molecular weight. SDS-PAGE gel confirmed the high purity of all the constructs purified using the nickel mag- netic beads (Fig. S4A). The migration pattern of the fusion OBPs do not correspond to the theoretical molecular weight of the proteins. OBP:pVEC (22.3 kDa) runs faster in SDS-PAGE than OBP:Tat $(21.6 \mathrm{kDa})$. As other authors reported, proteins can in some cases display anomalous migration in SDS-PAGE relatively to protein standards. Other techniques such as mass spectrometry, like MALDI-TOF, are more appropriate to precisely measure the mass of the protein [37,38].

Data obtained by MALDI-TOF confirmed the monodispersity of purified proteins whereas the experimental molecular weight is in accordance with the theoretical values (Fig. S4B).

\subsection{Structural analysis by CD spectroscopy}

The effect of CPPs on OBP structure was evaluated by circular dichroism (CD) spectroscopy. OBP-I belongs to lipocalin superfamily that is known to share a conserved folding pattern, an eight stranded $\beta$-barrel flanked by a $\alpha$-helix at the C-terminal end of the polypeptide chain [9]. The shape of the spectra of OBP wt, OBP:Tat and OBP:Pep-1 is similar, i.e., the maximum and minimum, respectively, at $195 \mathrm{~nm}$ and $215 \mathrm{~nm}$, are characteristic of a fold with a high content of $\beta$-sheets (Fig. 3A). The fusion of OBP with Tat and Pep-1 did not disturb the protein conformation. The spectrum of OBP:pVEC (Fig. 3A, dash line) displayed less pronounced peaks. This loss in intensity indicates a more extended state of the $\beta$-sheets resulting from the partial unfolding of OBP promoted by the pVEC peptide $[39,40]$. The presence of 1-AMA did not significantly change the OBP:CPPs spectra as shown in Fig. 3C. The differences detected are related with a protein rearrangement associated with the binding of the ligand to the aromatic residues of the OBP pocket $[15,41]$. CPPs alone showed a negative band at 195-205 nm characteristic of a random coil conformation (Fig. 3B). This result is expectable for CPPs when in aqueous buffers. Nevertheless, these peptides can acquire other conformations in the presence of different solvents [17].

\subsection{Binding capacity of fusion proteins}

The binding ability of OBP:CPPs was evaluated by fluorescence binding assay using 1-AMA as ligand model. When 1-AMA is at the binding site of OBP-I, the emission wavelength undergoes a minor blue shift and the intensity of the fluorescence is greatly increased [9]. The free 1-AMA is monitored at $537 \mathrm{~nm}$ while the binding of 1 -AMA to OBP is measured at $481 \mathrm{~nm}\left(\lambda_{\mathrm{ex}}=295 \mathrm{~nm}\right)$. Binding strength, measured in terms of the dissociation constant (Kd), revealed different behavior for the three OBP:CPPs (Fig. 4). Binding curves obtained by fluorescence reveal similar dissociations constants for OBP:Pep-1 $(\mathrm{Kd}=0.56 \mu \mathrm{M})$, OBP:Tat $(\mathrm{Kd}=0.58 \mu \mathrm{M})$ and OBP wt $(\mathrm{Kd}=0.44 \mu \mathrm{M})$ while a distinct behavior was observed for OBP:pVEC $(\mathrm{Kd}=2.46 \mu \mathrm{M})$. The high dissociation value of OBP:pVEC fusion protein could be related with the structural rearrangements 
A

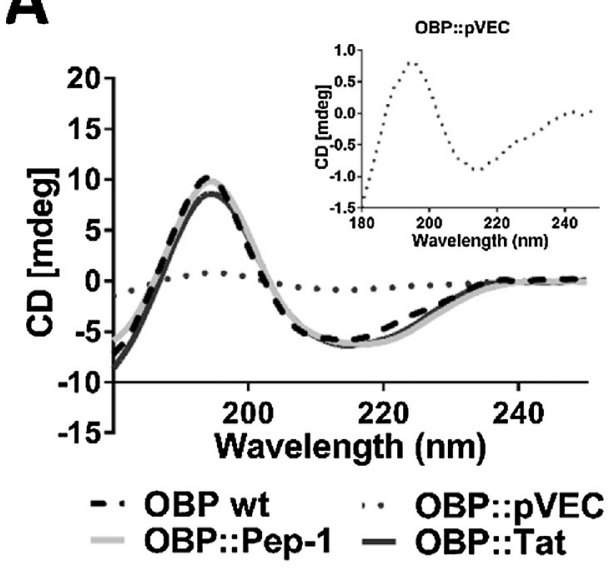

C
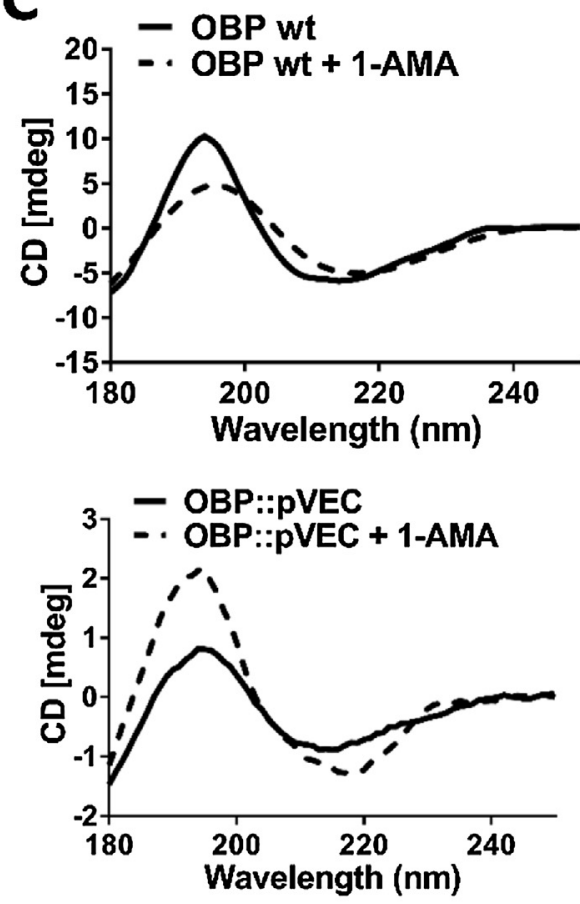

B
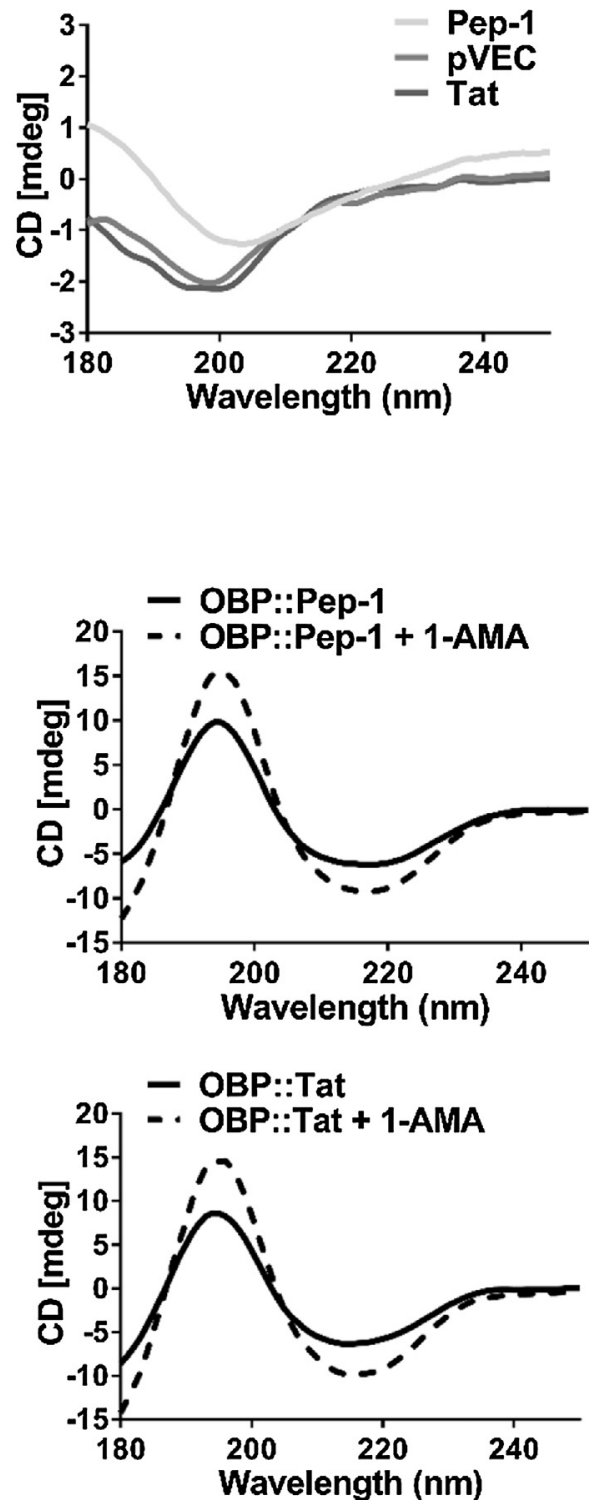

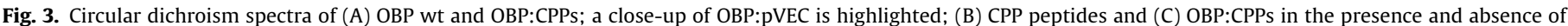
1-AMA; for a better visualization of CD spectrum of CPPs and OBP:pVEC different scales were used.

induced by the presence of the CPP, as indicated by CD spectra in Fig. 3A.

\subsection{Transduction of 1-AMA driven by OBP:CPPs into liposomes reservoirs}

A new methodology was developed to monitor 1-AMA transduction into liposomes membrane (Fig. 2). Non-toxic and biodegradable [42] liposomes were used as reservoirs of 1-AMA transduced by OBP:CPPs. To optimize the 1-AMA transduction efficiency, several incubation periods were tested (data not shown). We observed that $1 \mathrm{~h}$ of incubation at step 1 (OBP:CPPs + 1-AMA) and $1 \mathrm{~h}$ of incubation at step 2 (OBP:CPPs/1-AMA complex + liposomes) resulted in higher transduction efficiencies. The results obtained (Table 2) revealed the role of $\mathrm{OBP}: \mathrm{CPP}$ construct on the transduction efficiency of 1-AMA. OBP:Tat presented a transduction efficiency near $42 \%$, whereas OBP:Pep-1 and OBP:pVEC, showed efficiencies of 31.6 and $17.2 \%$, respectively. These differences can be attributed to the amino acid sequence of CPPs, their charge, and hydropho-
Table 2

1-AMA transduction efficiency driven by OBP:CPPs into liposomes and 1-AMA binding dissociation constants of OBP:CPPs; the CPP effect was determined relatively to the OBP wt. Values are the mean \pm SD of 2 independent experiments.

\begin{tabular}{llll}
\hline Protein & Kd $(\mu \mathrm{M})$ & Efficiency $(\%)$ & CPP effect $(\%)$ \\
\hline OBP:Tat & 0.58 & $41.93 \pm 0.73$ & 16.50 \\
OBP:Pep-1 & 0.56 & $31.64 \pm 2.36$ & 6.20 \\
OBP wt & 0.44 & $25.47 \pm 0.16$ & - \\
OBP:pVEC & 2.46 & $17.27 \pm 0.32$ & $\approx 0$ \\
\hline
\end{tabular}

bic/hydrophilic character, and to the structural conformation of OBP [43]. Peptides rich in arginine residues $(R)$ like Tat, have been described as having higher transduction ability than CPPs rich in lysine residues (K), like Pep-1, and than peptides rich in leucine (L) and isoleucine (I) residues, like pVEC [44]. According to the work of Herce et al. (2009), the arginine and lysine residues of Tat initially bind to the phosphate groups of phospholipids producing a strong alteration of the membrane. A pore is formed due to translocation of a single arginine residue to the phosphate group of DOPC [45]. 


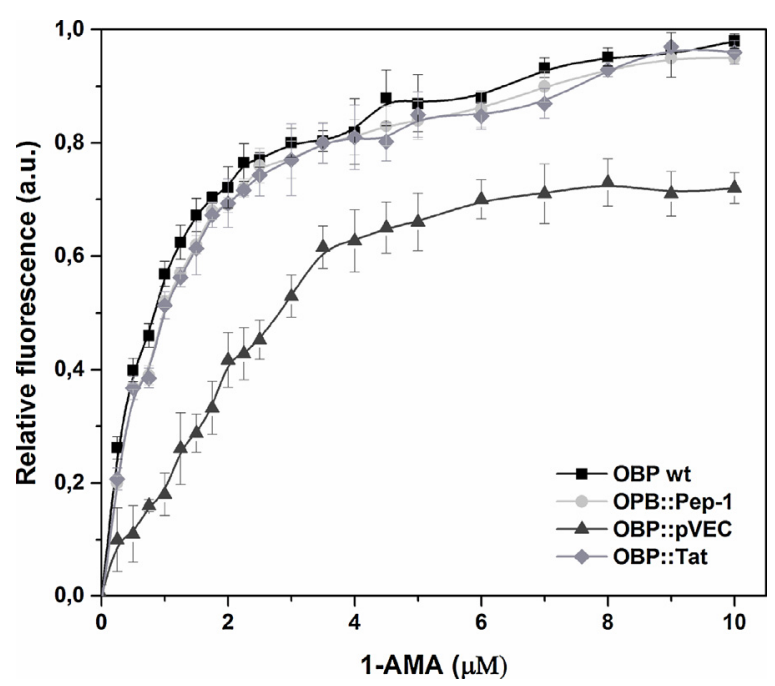

Fig. 4. Binding curves obtained by measuring the fluorescence of $1 \mu \mathrm{M}$ OBPs:CPPs in $50 \mathrm{mM}$ Tris- $\mathrm{HCl}, \mathrm{pH} 7.5$, at equilibrium with several concentrations of 1-aminoanthracene. The dissociation constants were obtained at $37^{\circ} \mathrm{C}$ by mathematical fitting of data [49]. Values are the mean \pm SD of 3 independent experiments.

Recent work of Nischan et al. (2015) showed the transduction efficiency of cyclic Tat conjugated with green fluorescent protein (GFP) into the cytosol of living cells and compare it with an amphipathic peptide, PTD4 [46]. They observed that while Tat was successfully delivered into the cell, PTD4 was retained on the endosomes [46].

Higher transduction efficiency of 1-AMA by OBP:Tat can also be associated to the high pKa of arginine which results on the delocalization of the positive charge of guanidinium side chain promoting the protonation of arginine even within membranes. This charge delocalization might contribute to the destabilization of the liposomes membrane [45].

The hydrophilicity of CPPs plays also an important role on transduction efficiency of 1-AMA. According to Expasy ProtParam tool, the grand average of hydropathicity (GRAVY) indicated that the three CPPs are hydrophilic, being Tat $(-3.492)$ the more hydrophilic followed by Pep-1 (-2.038) and pVEC (-0.444) [47]. As more hydrophilic CPP is, higher levels of transduction are obtained [48]. The lower transduction efficiency obtained by OBP:pVEC is associated to the destabilization of OBP structural conformation induced by the CPP, as confirmed by CD spectra (Fig. 3A).

The binding capacity of CPPs to 1-AMA was evaluated (data not shown) to confirm the role of OBP on the binding event. A residual fluorescence signal at $481 \mathrm{~nm}$ was detected indicating that 1-AMA is not binding to CPPs. 1-AMA in the presence of liposomes was performed as a control on the liposomal transduction experiments. This control revealed no 1-AMA transduction.

Liposomes were physicochemical characterized by means of size, polydispersity index, and surface charge after transduction. Liposomes maintain the mean size with a small variation of the surface charge, being stable for at least 2 months of storage (Table S1).

The prediction of 1-AMA distribution in the system was obtained by fluorescence spectra using the same conditions described previously (Fig. S5). Maximum peaks were determined when 1-AMA is free, bind to the protein and transduced into liposomes. The deconvolution of these peaks allowed to determine the percentage of 1-AMA distribution (Table S2) which was in accordance with the experimental transduction efficiency data obtained (Table 2).

After transduction we evaluated the extent of interaction between liposomes and OBP:CPPs. A considerable amount of OBP:CPPs appears to be near by the liposome bilayer, up to $56 \%$. We further check the possibility of OBP:CPPs internalization by liposomes model (MLVs) and the protein signal was mostly found outside of MLVs (Fig. S6).

\subsection{Free energy calculations on 1-AMA transduction}

The potentials of mean force (PMF) calculations were performed to access the transduction of 1-AMA by OBP wt. The free energy profile of 1-AMA transduced through the bilayer, obtained by umbrella sampling technique and WHAM analysis, is shown in Fig. 5. The binding site of the protein corresponds to $0 \mathrm{~nm}$ (start point) in the $\mathrm{z}$ direction.

The PMF curve showed above indicates the probable distribution/behavior of 1-AMA at different regions of the system: complexed with the OBP, in the water layer, at the lipid head groups or in the interior of the membrane. According to the results obtained with PMF calculations, the most favored locations of 1AMA are the internal cavity of OBP wt or inside the membrane (in the middle of the bilayer). We can observe the occurrence of a high energy barrier to unbind the 1-AMA from OBP, probably due to hydrogen bond loss at the complexed state, and from water layer to bilayer surface, as expected once the ligand is hydrophobic, showing repulsion when in these two polar environments. Qualitatively, we observed that 1-AMA encounter a cavity suitable for insertion while moving across the bilayer surface, and the membrane designed and equilibrated with our developed parameters, readjust without disorder to receive the ligand.

The free energy profile indicates that 1-AMA can penetrate this model membrane when forming a complex with OBP wt. More important, we can predict that the closer the protein is to the membrane, promoted by the presence of CPPs the lower will be the energy barrier. The gap between the protein and the membrane is decreased by the presence of the CPPs.

\section{Conclusions}

OBP intrinsic binding properties together with CPPs ability to penetrate into lipid membranes were explored for the capture and transduction of 1-AMA into liposome reservoirs. We reported for the first time the fusion of pig OBP with three cell penetrating peptides (Tat, pVEC and Pep-1). No structural changes of OBP were detected by circular dichroism spectroscopy after fusion with these peptides, except for pVEC. High binding affinity towards 1-AMA was observed for OBP:Pep-1 and OBP:Tat, whereas OBP:pVEC, due to the protein structural changes induced by the pVEC sequence, resulted in lower binding affinity. The transduction of 1-AMA driven by OBP:CPPs into liposomes is governed by the CPP amino acidic sequence, by their hydrophobic/hydrophilic character and by their charge. The highest 1-AMA transduction efficiency was obtained for OBP:Tat fusion protein.

The new approach including OBP:CPP fusion proteins together with liposomes, as tailored reservoirs, open up new opportunities for the development of controlled systems to be used in a wide range of applications, namely for textiles functionalization, as biosensors, among others.

\section{Acknowledgements}

This study was supported by the Portuguese Foundation for Science and Technology (FCT) under the scope of the strategic funding of UID/BIO/04469/2013 unit and COMPETE 2020 (POCI-01-0145-FEDER-006684). Artur Ribeiro and Filipa Gonçalves thank FCT for funding their scholarships with the references SFRH/BPD/98388/2013and SFRH/BD/114684/2016, respectively. Tarsila G. Castro thanks the senior position funded by the European Union through the European Regional Development Fund (ERDF) 


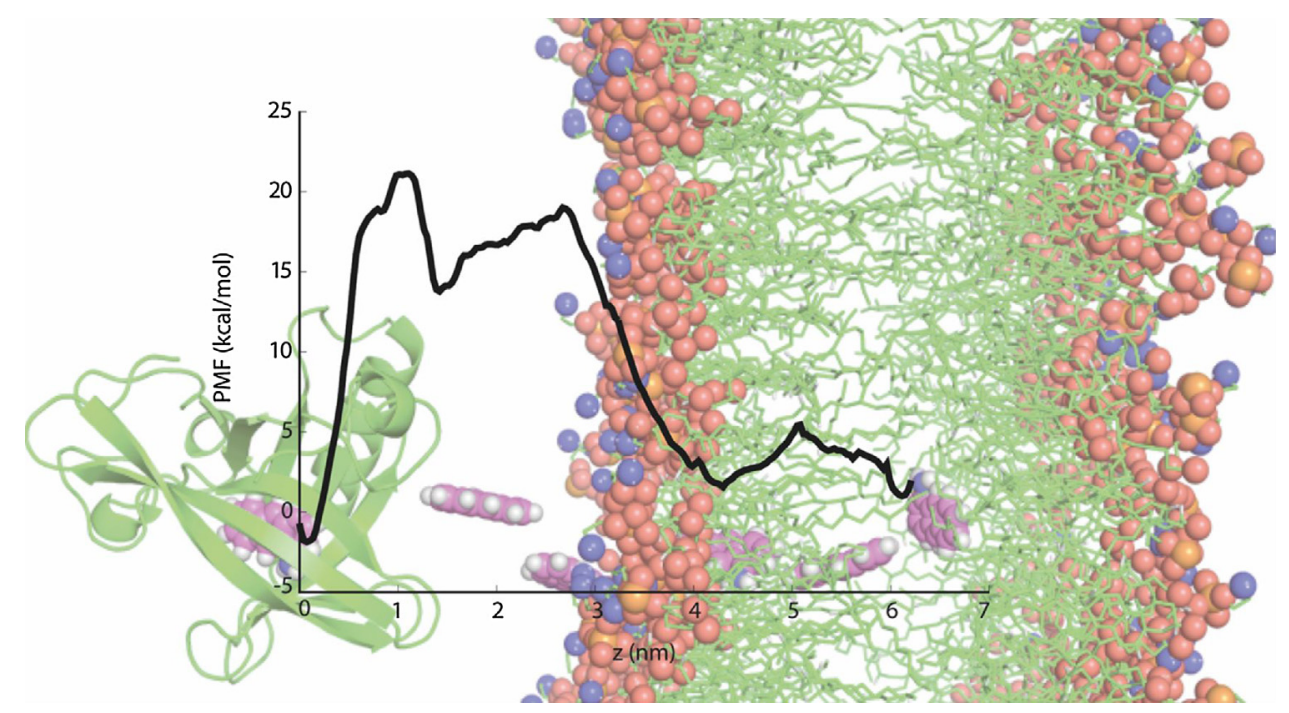

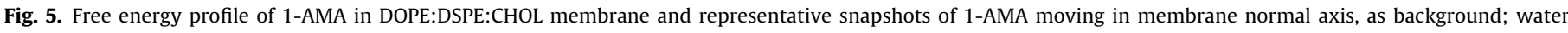

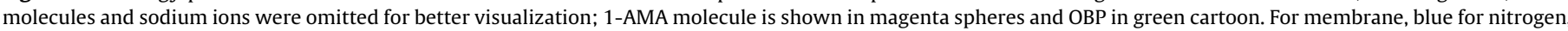


article.)

under the Competitiveness Operational Program (COP-A1-A1.1.4-E nr.30/01.09.2016).

Access to computing resources funded by the Project "SearchON2: Revitalization of HPC infrastructure of UMinho" (NORTE-070162-FEDER-000086), co-funded by the North Portugal Regional Operational Program (ON.2-O Novo Norte), under the National Strategic Reference Framework (NSRF), through the European Regional Development Fund (ERDF), is also gratefully acknowledged.

\section{Appendix A. Supplementary data}

Supplementary data associated with this article can be found, in the online version, at https://doi.org/10.1016/j.colsurfb.2017.11. 026.

\section{References}

[1] B. Hess, C. Kutzner, D. van der Spoel, E. Lindahl, GROMACS 4: algorithms for highly efficient, load-Balanced, and scalable molecular simulation, J. Chem. Theory. Comput. 4 (2008) 435-447.

[2] Z. Guo, H. Peng, J. Kang, D. Sun, Cell-penetrating peptides: possible transduction mechanisms and therapeutic applications, Biomed. Reports 4 (2016) 528-534.

[3] A. El-Sayed, S. Futaki, H. Harashima, Delivery of macromolecules using arginine-rich cell-penetrating peptides: ways to overcome endosomal entrapment, AAPS J. 11 (2009) 13-22.

[4] S.B. Fonseca, M.P. Pereira, S.O. Kelley, Recent advances in the use of cell-penetrating peptides for medical and biological applications, Adv. Drug Deliv. Rev. 61 (2009) 953-964.

[5] A. Elmquist, M. Lindgren, T. Bartfai, U. Langel, VE-cadherin-derived cell-penetrating peptide pVEC, with carrier functions, Exp. Cell Res. 269 (2001) 237-244.

[6] H. Jing, W. Cheng, S. Li, B. Wu, X. Leng, S. Xu, J. Tian, Novel cell-penetrating peptide-loaded nanobubbles synergized with ultrasound irradiation enhance EGFR siRNA delivery for triple negative Breast cancer therapy, Colloids Surf. B Biointerfaces 146 (2016) 387-395.

[7] R. Mastrogiacomo, I. Iovinella, E. Napolitano, New fluorescent probes for ligand-binding assays of odorant-binding proteins, Biochem. Biophys. Res. Commun. 446 (2014) 137-142.

[8] M.Y. Mulla, E. Tuccori, M. Magliulo, G. Lattanzi, G. Palazzo, K. Persaud, L. Torsi, Capacitance-modulated transistor detects odorant binding protein chiral interactions, Nat. Commun. 6 (2015) 6010.

[9] S. Paolini, F. Tanfani, C. Fini, E. Bertoli, P. Pelosi, Porcine odorant-binding protein: structural stability and ligand affinities measured by fourier-transform infrared spectroscopy and fluorescence spectroscopy, Biochim. et Biophys. Acta 1431 (1999) 179-188.
[10] Y. Wei, A. Brandazza, P. Pelosi, Binding of polycyclic aromatic hydrocarbons to mutants of odorant-binding protein: a first step towards biosensors for environmental monitoring, Biochim. Biophys. Acta 1784 (2008) 666-671.

[11] F. Di Pietrantonio, D. Cannata, M. Benetti, E. Verona, A. Varriale, M. Staiano, S. D'Auria, Detection of odorant molecules via surface acoustic wave biosensor array based on odorant-binding proteins, Biosens. Bioelectron. 41 (2013) 328-334.

[12] C. Silva, T. Matama, N.G. Azoia, C. Mansilha, M. Casal, A. Cavaco-Paulo, Odorant binding proteins: a biotechnological tool for odour control, Appl. Microbiol. Biotechnol. 98 (2014) 3629-3638.

[13] C. Bechara, S. Sagan, Cell-penetrating peptides: 20 years later where do we stand? FEBS Lett. 587 (2013) 1693-1702.

[14] E. Gros, S. Deshayes, M.C. Morris, G. Aldrian-Herrada, J. Depollier, F. Heitz, G. Divita, A non-covalent peptide-based strategy for protein and peptide nucleic acid transduction, Biochim. Biophys. Acta 1758 (2006) 384-393.

[15] D. Kmiecik, J.R. Albani, Effect of 1-aminoanthracene (1-AMA) binding on the structure of three lipocalin proteins the dimeric beta lactoglobulin, the dimeric odorant binding protein and the monomeric alpha1-acid glycoprotein. Fluorescence spectra and lifetimes studies, J. Fluoresc. 20 (2010) 973-983.

[16] <Briand et al., 2000 - Odorant-binding protein variant.pdf>

[17] E. Eiriksdottir, K. Konate, U. Langel, G. Divita, S. Deshayes, Secondary structure of cell-penetrating peptides controls membrane interaction and insertion, Biochim. Biophys. Acta 1798 (2010) 1119-1128.

[18] <brunori1983. pdf>.

[19] E. Nogueira, I.C. Mangialavori, A. Loureiro, N.G. Azoia, M.P. Sarria, P. Nogueira, J. Freitas, J. Harmark, U. Shimanovich, A. Rollett, G. Lacroix, G.J. Bernardes, G. Guebitz, H. Hebert, A. Moreira, A.M. Carmo, J.P. Rossi, A.C. Gomes, A. Preto, A. Cavaco-Paulo, Peptide anchor for folate-Targeted liposomal delivery, Biomacromolecules 16 (2015) 2904-2910.

[20] H.M. Berman, J. Westbrook, Z. Feng, G. Gilliland, T.N. Bhat, H. Weissig, I.N. Shindyalov, P.E. Bourne, The protein data bank, Nucleic Acids Res. 28 (2000) $235-242$.

[21] F. Vincent, S. Spinelli, R. Ramoni, S. Grolli, P. Pelosi, C. Cambillau, M. Tegoni, Complexes of porcine odorant binding protein with odorant molecules belonging to different chemical classes1, J. Mol. Biol. 300 (2000) 127-139.

[22] Schrödinger, The PyMOL Molecular Graphics System, LLC, 2010.

[23] D. v. d. Spoel, E. Lindahl, B. Hess, A.R. v. Buuren, E. Apol, P.J. Meulenhoff, P. Tieleman, A.L.T.M. Sjibers, K.A. Feenstra, R. v. Drunen, H.J.C. Berendsen, Gromacs User Manual Version 4.5, 2010.

[24] W. Huang, Z.X. Lin, W.F. van Gunsteren, Validation of the GROMOS 54A7 force field with respect to beta-Peptide folding, J. Chem. Theory. Comput. 7 (2011) 1237-1243

[25] T. Darden, D. York, L. Pedersen, Particle mesh ewald - an n.log(n) method for ewald sums in large systems, J. Chem. Phys. 98 (1993) 10089-10092.

[26] B. Hess, P-LINCS: A parallel linear constraint solver for molecular simulation, J. Chem. Theory. Comput. 4 (2008) 116-122.

[27] B. Hess, H. Bekker, H.J.C. Berendsen, J. Fraaije, LINCS: A linear constraint solver for molecular simulations, J. Comput. Chem. 18 (1997) 1463-1472.

[28] D. van der Spoel, P.J. van Maaren, H.J.C. Berendsen, A systematic study of water models for molecular simulation: derivation of water models optimized for use with a reaction field, J. Chem. Phys. 108 (1998) 10220-10230. 
[29] H.J.C. Berendsen, J.P.M. Postma, W.F. Vangunsteren, A. Dinola, J.R. Haak, Molecular-dynamics with coupling to an external bath, J. Chem. Phys. 81 (1984) 3684-3690.

[30] D.J. Evans, B.L. Holian, The nose-hoover thermostat, J. Chem. Phys. 83 (1985) 4069-4074

[31] W.G. Hoover, Canonical dynamics: equilibrium phase-space distributions, Phys. Rev. A 31 (1985) 1695-1697.

[32] R. Martonak, A. Laio, M. Parrinello, Predicting crystal structures: the Parrinello-Rahman method revisited, Phys. Rev. Lett. 90 (2003) 4.

[33] S. Kumar, J.M. Rosenberg, D. Bouzida, R.H. Swendsen, P.A. Kollman, THE weighted histogram analysis method for free-energy calculations on biomolecules I. The method, J. Comp.Chem. 13 (1992) 1011-1021.

[34] M.R. Shirts, D.L. Mobley, An introduction to best practices in free energy calculations, in: L. Monticelli, E. Salonen (Eds.), Biomolecular Simulations: Methods and Protocols, Humana Press, Totowa, NJ, 2013, pp. 271-311.

[35] B. Roux, The calculation of the potential of mean force using computer simulations, Comput. Phys. Commun. 91 (1995) 275-282.

[36] J.S. Hub, B.L. de Groot, D. van der Spoel, G_wham-A free weighted histogram analysis implementation including robust error and autocorrelation estimates, J. Chem. Theory Comput. 6 (2010) 3713-3720.

[37] W. Hassouneh, S.R. MacEwan, A. Chilkoti, Fusions of elastin-like polypeptides to pharmaceutical proteins, Methods Enzymol. 502 (2012) 215-237.

[38] A. Matagne, B. Joris, J.-M. Frére, Anomalous behaviour of a protein during SDS/PAGE corrected by chemical modification of carboxylic groups, Biochem. J. 280 (1991) 553-556

[39] G. Manzo, M.A. Scorciapino, P. Wadhwani, J. Burck, N.P. Montaldo, M. Pintus, R. Sanna, M. Casu, A. Giuliani, G. Pirri, V. Luca, A.S. Ulrich, A.C. Rinaldi, Enhanced amphiphilic profile of a short beta-stranded peptide improves its antimicrobial activity, PLoS One 10 (2015) e0116379.

[40] J.C. Ioannou, A.M. Donald, R.H. Tromp, Characterising the secondary structure changes occurring in high density systems of BLG dissolved in aqueous $\mathrm{pH} 3$ buffer, Food Hydrocolloids 46 (2015) 216-225.
[41] Y.A. Nagel, P.S. Raschle, H. Wennemers, Effect of preorganized charge-Display on the cell-Penetrating properties of cationic peptides, Angew. Chem. Int. Ed. Eng. 56 (2017) 122-126.

[42] G. Bozzuto, A. Molinari, Liposomes as nanomedical devices, Int. J. Nanomed. 10 (2015) 975-999.

[43] S. Deshayes, M.C. Morris, G. Divita, F. Heitz, Cell-penetrating peptides: tools for intracellular delivery of therapeutics, Cell. Mol. Life Sci. 62 (2005) 1839-1849.

[44] I. Nakase, T. Takeuchi, G. Tanaka, S. Futaki, Methodological and cellular aspects that govern the internalization mechanisms of arginine-rich cell-penetrating peptides, Adv. Drug Deliv. Rev. 60 (2008) 598-607.

[45] J. Ahnstrom, K. Faber, O. Axler, B. Dahlback, Hydrophobic ligand binding properties of the human lipocalin apolipoprotein M, J. Lipid Res. 48 (2007) 1754-1762.

[46] N. Nischan, H.D. Herce, F. Natale, N. Bohlke, N. Budisa, M.C. Cardoso, C.P. Hackenberger, Covalent attachment of cyclic TAT peptides to GFP results in protein delivery into live cells with immediate bioavailability, Angew. Chem. Int. Ed. Eng. 54 (2015) 1950-1953.

[47] E. Gasteiger, C. Hoogland, A. Gattiker, S. Duvaud, M. Wilkins, R. Appel, A. Bairoch, Protein identification and analysis tools on the ExPASy server, in: J.M. Walker (Ed.), The Proteomics Protocols Handbook, Humana Press, 2005, pp. 571-607.

[48] H.D. Herce, A. Garcia, J. Litt, P. Martin, N. Enrique, A. Rebolledo, V. Milesi, Arginine-rich peptides destabilize the plasma membrane, consistent with a pore formation translocation mechanism of cell penetrating peptides, Biophys. J. 7 (2009) 1917-1925.

[49] G. Malpeli, C. Folli, D. Cavazzino, G. Sartori, R. Berni, Purification and fluorescent titration of cellular retinol-Binding protein, in: C.P.F. Redfern (Ed.), Methods in Molecular Biology, Springer International Publishing, Totowa, NJ, 1998. 